\title{
A Simultaneous Search Problem ${ }^{1}$
}

\author{
E.-C. Chang ${ }^{2,3}$ and C. Yap ${ }^{2}$
}

\begin{abstract}
We introduce a new search problem motivated by computational metrology. The problem is as follows: we would like to locate two unknown numbers $x, y \in[0,1]$ with as little uncertainty as possible, using some given number $k$ of probes. Each probe is specified by a real number $r \in[0,1]$. After a probe at $r$, we are told whether $x \leq r$ or $x \geq r$, and whether $y \leq r$ or $y \geq r$. We derive the optimal strategy and prove that the asymptotic behavior of the total uncertainty after $k$ probes is $\frac{13}{7} 2^{-(k+1) / 2}$ for odd $k$ and $\frac{13}{10} 2^{-k / 2}$ for even $k$.
\end{abstract}

Key Words. Algorithm, Binary search, Probe model, Comparison model, Metrology.

1. Introduction. The following search problem was introduced by [4] in the context of geometric tolerancing and metrology [2], [1], [3]. Given a closed interval $B \subseteq \mathbb{R}$, our task is to estimate its length $L=|B|$. In practice, $B$ is a rod or some body whose length we wish to estimate. Toward this end, we are to probe $B$ using a grid which, after a scaling factor, may be identified with $\mathbb{Z}$. The initial probe amounts to placing $B$ arbitrarily on the real line-if a placement is specified by a real number $s_{0} \in \mathbb{R}$, then the position of $B$ in placement $s_{0}$ corresponds to the interval $B+s_{0}=\left\{x+s_{0}: x \in B\right\}$. See Figure 1 for an illustration.

The result of the initial probe is the discrete set

$$
S_{0}:=\left(B+s_{0}\right) \cap \mathbb{Z}
$$

In Figure 1, $S_{0}$ has five points. It is immediate that if $n_{0}=\left|S_{0}\right|$, then

$$
\left(n_{0}-1\right) \leq L<\left(n_{0}+1\right)
$$

So the uncertainty about $L$ is 2 after the initial probe.

In subsequent probes, we are allowed to shift $B$ by any desired amount. If the first probe after the initial probe is obtained by shifting $B$ by $s_{1}$, then $B$ is next placed in position $B+s_{0}+s_{1}$, and the result of this probe is the set

$$
S_{1}:=\left(B+s_{0}+s_{1}\right) \cap \mathbb{Z} .
$$

\footnotetext{
${ }^{1}$ This research was supported in part by NSF Grant \#CCR-9619846 and by NATO Grant \#CRG-950367.

${ }^{2}$ Courant Institute of Mathematical Sciences, New York University, 251 Mercer Street, New York, NY 10012, USA.

${ }^{3}$ Current address: Department of Computational Science, National University of Singapore, 10 Kent Ridge Crescent, Singapore 11926.
}

Received November 11, 1996; revised October 2, 1997, and July 13, 1998. Communicated by D. P. Dobkin. 


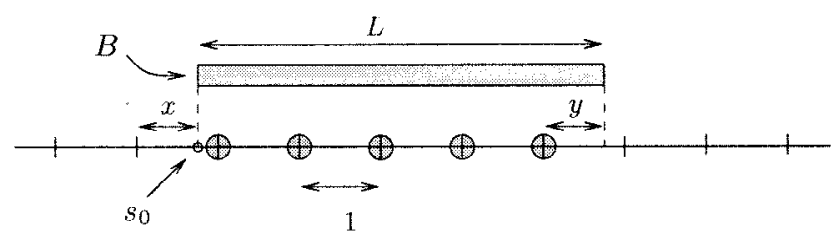

Fig. 1. A $\operatorname{rod} B$ at position $s_{0}$ on a grid.

To ensure that $S_{1}$ is nonempty, we assume $L>1$. In general, if the $k$ th shift is $s_{k}$, then the result of the corresponding probe is the set

$$
S_{k}:=\left(B+\sum_{i=0}^{k} s_{i}\right) \cap \mathbb{Z} .
$$

For any given $k \geq 0$, our goal is to devise a strategy of choosing $k$ shifts so that the worst case uncertainty concerning $L$ is minimized. It is not hard to see that we may restrict $s_{i}$ so that $0<s_{i}<1$.

2. The Abstract Problem. We reformulate the above problem in an abstract setting. To establish the context, recall the classic problem of searching for an unknown real number $x$, known to lie in some interval $I_{0} \subseteq \mathbb{R}$. We are allowed to compare $x$ with any chosen real number $r \in \mathbb{R}$. Such a comparison, denoted $x: r$, has one of two possible outcomes " $x \leq r$ " or " $x \geq r$." The classic binary search algorithm, after making $k$ comparisons, determines a subinterval $I_{k} \subseteq I_{0}$ of size $\left|I_{k}\right|=2^{-k}\left|I_{0}\right|$. Interpreting $\left|I_{k}\right|$ as the uncertainty of $x$ after $k$ comparisons, it is well known that the binary search algorithm is optimal, that is, it achieves the minimax uncertainty after $k$ comparisons.

Now consider a generalization called a simultaneous searching problem: we are given two intervals $I, J \subseteq \mathbb{R}$ and a number $k \geq 0$. Our goal is to locate two unknown numbers $x \in I$ and $y \in J$ as accurately as possible using $k$ probes. Each probe is specified by a real number $r \in \mathbb{R}$ called the discriminant, and it corresponds to making a pair of simultaneous comparisons, $x: r$ and $y: r$. If the outcome is $x \geq r$, then $I$ is next reduced to $I^{\prime}=I \cap\{\alpha \in \mathbb{R}: \alpha \geq r\}$ and otherwise $I^{\prime}=I \cap\{\alpha \in \mathbb{R}: \alpha \leq r\}$. The outcome of the comparison on $y$ is similarly treated, and let $J$ be updated to $J^{\prime}$. Notice that if $I \cap J=\emptyset$, then a probe amounts to a choice of one of the two intervals $I$ or $J$ upon which to perform an ordinary comparison.

The uncertainty of $I, J$ is given by $|I|+|J|$. After a probe, uncertainty is reduced to $\left|I^{\prime}\right|+\left|J^{\prime}\right|$. Let $U_{k}(I, J)$ denote the minimax uncertainty after $k$ probes. Let $\sigma_{k}(I, J)$ be the discriminant $r$ of the first probe in an optimal $k$-probe strategy. We are interested in two special cases:

DisJOINT CASE. This is when $I \cap J=\emptyset$. Clearly, $U_{k}(I, J)$ depends only on the lengths $\alpha=|I|$ and $\beta=|J|$. If $\alpha+\beta=1$, we write $V_{k}(\alpha)$ for $U_{k}(I, J)$.

JOINT CASE. This is when $I=J$. If $I=J=[0,1]$, we write $U_{k}$ and $\sigma_{k}$ instead of $U_{k}(I, J)$ and $\sigma_{k}(I, J)$, respectively. Hence $U_{0}=2$ and, by definition, $\sigma_{0}=0$. 
In our metrology problem to estimate the length $L$ of a $\operatorname{rod} B$, we began with an initial probe (Figure 1). Let $x$ (respectively $y$ ) be the distance of the rod's left (respectively right) end to the nearest grid point on the left. Clearly $x, y \in[0,1]$. Thus $x$ and $y$ correspond to the unknown numbers of the abstract problem with $I=J=[0,1]$. In general, after the $i$ th probe $(i=0,1, \ldots, k)$, the left and right endpoints of $B$ can be located within two intervals $I_{i}, J_{i}$ which can be specified as follows. Let $S_{i}=$ $\left(B+\sum_{j=0}^{k} s_{i}\right) \cap \mathbb{Z}$ be the result of the $i$ th probe as in the Introduction. If $S_{i}$ comprises the integers $m_{i}, m_{i}+1, \ldots, n_{i}-1, n_{i}$, then it is sufficient to specify the intervals $\hat{I}_{i}$ and $\hat{J}_{i}$ which relate to $I_{i}$ and $J_{i}$ via the equations $I_{i}=m_{i}-1+\hat{I}_{i}$ and $J_{i}=n_{i}+\hat{J}_{i}$. Initially, $\hat{I}_{0}=\hat{J}_{0}=[0,1]$. For $i \geq 1$,

$$
\hat{I}_{i}= \begin{cases}\left(s_{i}+\hat{I}_{i-1}\right) \cap[0,1] & \text { if } \quad m_{i}=m_{i-1}, \\ \left(s_{i}-1+\hat{I}_{i-1}\right) \cap[0,1] & \text { if } \quad m_{i}=m_{i-1}+1,\end{cases}
$$

and similarly for $\hat{J}_{i}$. It is easy to see that $x \in I_{i}-\left(\sum_{j=1}^{i} s_{j}\right)-\left(m_{0}-1\right)$, and $y \in$ $J_{i}-\left(\sum_{j=1}^{i} s_{j}\right)-n_{0}$, so that $\left|\hat{I}_{i}\right|+\left|\hat{J}_{i}\right|$ is the uncertainty about the numbers $x, y$ after the $i$ th probe. The $i$ th probe corresponds to the comparisons $x: r_{i}$ and $y: r_{i}$, where $r_{i}=\left(-\sum_{j=1}^{i} s_{j}\right) \bmod 1$.

It is not hard to see that $U_{1}=1$. Next we claim that

$$
U_{2}=\frac{2}{3}
$$

To see that $U_{2} \leq \frac{2}{3}$, let the discriminant of the first probe be $\frac{1}{3}$. There are basically two cases of the resultant intervals $\left(I^{\prime}, J^{\prime}\right)$ to consider: $\left(I^{\prime}, J^{\prime}\right)=\left(\left[\frac{1}{3}, 1\right],\left[\frac{1}{3}, 1\right]\right)$ or $\left(I^{\prime}, J^{\prime}\right)=\left(\left[0, \frac{1}{3}\right],\left[\frac{1}{3}, 1\right]\right)$. In either case, the discriminant of the next probe (second probe) can be chosen as $\frac{2}{3}$. We see that the uncertainty is at most $\frac{2}{3}$ after this probe. To see that $U_{2} \geq \frac{2}{3}$, suppose the first probe discriminant is $r \neq \frac{1}{3}$. If $r>\frac{1}{3}$, then $U_{2} \geq U_{1}([0, r],[r, 1])>\frac{2}{3}$; otherwise $r<\frac{1}{3}$ and we have $U_{2} \geq U_{1}([r, 1],[r, 1])>\frac{2}{3}$.

We have the following bound for any $|I|=|J|=1$ :

$$
2^{1-k} \leq U_{k}(I, J) \leq 2^{1-\lfloor k / 2\rfloor} .
$$

The lower bound of $U_{k}$ comes from the fact that each probe reduces the uncertainty by a factor of at most $\frac{1}{2}$. The upper bound on $U_{k}$ comes from the fact that we can reduce the uncertainty by a factor of at least $\frac{1}{2}$ with every two probes.

The main result of this paper determines the behavior of $U_{k}$ as $k \rightarrow \infty$. To understand this behavior, we first normalize $U_{k}$ by defining

$$
u_{k}:=U_{k} 2^{\lceil k / 2\rceil} \text {. }
$$

Table 1 lists the initial values of $U_{k}$ and $\sigma_{k}$, separated into two parts depending on the parity of $k$. These values are computed by a procedure described in Section 4. It turns out that the sequence $\left\{u_{k}\right\}_{k=1}^{\infty}$ does not converge but has two limits, depending on whether $k$ is even or odd:

$$
u_{2 k} \rightarrow \frac{13}{10}, \quad u_{2 k-1} \rightarrow \frac{13}{7} .
$$

This can be seen in Table 1 as well. 
Table 1. $\sigma_{k}$ and $U_{k}$.

\begin{tabular}{|c|c|c|}
\hline$k$ & $\sigma_{k}$ & $U_{k}$ \\
\hline 1 & $\frac{1}{2}=0.5$ & $1=2^{-1}(2)$ \\
\hline 3 & $\frac{5}{17}=0.2941 \ldots$ & $\frac{8}{17}=2^{-2}(1.8823 \ldots)$ \\
\hline 5 & $\frac{79}{275}=0.2872 \ldots$ & $\frac{64}{275}=2^{-3}(1.8618 \ldots)$ \\
\hline 7 & $\frac{1261}{4409}=0.2860 \ldots$ & $\frac{512}{4409}=2^{-4}(1.8580 \ldots)$ \\
\hline$\infty$ & $\frac{2}{7}=0.28571 \ldots$ & $\frac{13}{7} 2^{-(k+1) / 2}=2^{-(k+1) / 2}(1.8571 \ldots)$ \\
\hline 2 & $\frac{1}{3}=0.3333 \ldots$ & $\frac{2}{3}=2^{-1}(1.3333 \ldots)$ \\
\hline 4 & $\frac{15}{49}=0.3061 \ldots$ & $\frac{16}{49}=2^{-2}(1.3061 \ldots)$ \\
\hline 6 & $\frac{237}{787}=0.3011 \ldots$ & $\frac{128}{787}=2^{-3}(1.3011 \ldots)$ \\
\hline 8 & $\begin{array}{l}\frac{3783}{12601}=0.3002 \ldots \\
\vdots\end{array}$ & $\frac{1024}{12601}=2^{-4}(1.3002 \ldots)$ \\
\hline$\infty$ & $\frac{3}{10}=0.3$ & $\frac{13}{10} 2^{-k / 2}=2^{-k / 2}(1.3)$ \\
\hline
\end{tabular}

3. The Disjoint Case. Assume $I=[0, \alpha]$ and $J=[\alpha, 1]$. Let $V_{k}(\alpha):=U_{k}(I, J)$ be the minimax uncertainty for this particular setup. Observe that if $h$ probes are performed on the interval $I$, then the amount of uncertainty remaining in $I$ is $2^{-h} \alpha$. Thus,

$$
V_{k}(\alpha)=\min _{0<h \leq k}\left\{\frac{\alpha}{2^{h}}+\frac{1-\alpha}{2^{k-h}}\right\} .
$$

Normalize $V_{k}(\alpha)$ by considering the function

$$
v_{k}(\alpha):=2^{\lceil k / 2\rceil} V_{k}(\alpha) \text {. }
$$

For example, with $\alpha=\frac{1}{2}$, it is easy to see that $V_{k}\left(\frac{1}{2}\right)=2^{-k / 2}$ when $k$ is even and $V_{k}\left(\frac{1}{2}\right)=\frac{3}{2} 2^{-(k+1) / 2}$ when $k$ is odd. Hence $v_{k}\left(\frac{1}{2}\right)=1$ or 1.5 , depending on whether $k$ is even or odd. This behavior is seen generally in the next lemma.

LEMMA 1. Fix $0<\alpha \leq \frac{1}{2}$. As $k$ goes to infinity, the sequence $\left\{v_{k}(\alpha)\right\}_{k=1}^{\infty}$ does not converge but has two limit points. For even $k$ it converges to $v_{\mathrm{even}}(\alpha)$, whereas for odd $k$ it converges to $v_{\text {odd }}(\alpha)$, where

$$
\begin{aligned}
& v_{\text {even }}(\alpha)=2^{i} \alpha+2^{-i}(1-\alpha) \quad\left(\text { where } i=\left\lfloor\log _{4}(1-\alpha)-\log _{4} \alpha+\frac{1}{2}\right\rfloor\right) \\
& =\left\{\begin{array}{lll}
\alpha+1-\alpha & \text { if } & \frac{1}{2^{1}+1} \leq \alpha \leq \frac{1}{2}, \\
2 \alpha+\frac{1-\alpha}{2} & \text { if } \quad \frac{1}{2^{3}+1} \leq \alpha \leq \frac{1}{2^{1}+1}, \\
2^{2} \alpha+\frac{1-\alpha}{2^{2}} & \text { if } & \frac{1}{2^{5}+1} \leq \alpha \leq \frac{1}{2^{3}+1}, \\
& \ldots & \\
2^{i} \alpha+\frac{1-\alpha}{2^{i}} & \text { if } & \frac{1}{2^{2 i+1}+1} \leq \alpha \leq \frac{1}{2^{2(i-1)+1}+1}, \\
& \ldots &
\end{array}\right.
\end{aligned}
$$

and

$$
v_{\text {odd }}(\alpha)=2^{i} \alpha+2^{-i}(1-\alpha) \quad\left(\text { where } i=\left\lfloor\log _{4}(1-\alpha)-\log _{4} \alpha\right\rfloor\right)
$$




$$
=\left\{\begin{array}{lll}
2 \alpha+1-\alpha & \text { if } & \frac{1}{2^{2}+1} \leq \alpha \leq \frac{1}{2} \\
4 \alpha+\frac{1-\alpha}{2^{1}} & \text { if } & \frac{1}{2^{4}+1} \leq \alpha \leq \frac{1}{2^{2}+1} \\
8 \alpha+\frac{1-\alpha}{2^{2}} & \text { if } & \frac{1}{2^{6}+1} \leq \alpha \leq \frac{1}{2^{4}+1} \\
2^{i} \alpha+\frac{1-\alpha}{2^{i-1}} & \text { if } & \frac{1}{2^{2 i}} \leq \alpha \leq \frac{1}{2^{2 i-2}+1} \\
& \ldots &
\end{array}\right.
$$

ProOF. First assume $k$ is even and sufficiently large so that $\left(2^{k+1}+1\right)^{-1} \leq \alpha$. Let $I=$ $[0, \alpha]$ and $J=[\alpha, 1]$. For any positive integer $\ell \leq k / 2$, let $E_{\ell}(\alpha)=\alpha 2^{\ell}+(1-\alpha) 2^{-\ell}$. If we perform $(k / 2)-\ell$ comparisons in $I$ and the remaining $(k / 2)+\ell$ comparisons in $J$, then the remaining uncertainty is $2^{-\mathrm{k} / 2} E_{\ell}(\alpha)$. Observe that $v_{k}(\alpha)=\min _{\ell} E_{\ell}(\alpha)$. Writing $\alpha_{i}:=\left(2^{2 i+1}+1\right)^{-1}$, we may verify

$$
E_{i}\left(\alpha_{i}\right)=E_{i+1}\left(\alpha_{i}\right)
$$

We also note that

$$
\alpha<\alpha_{i} \quad \Longleftrightarrow \quad E_{i}(\alpha)>E_{i+1}(\alpha) .
$$

Thus $\alpha=\alpha_{i}$ is the cross-over point between optimally assigning $k / 2-i$ versus $k / 2-$ $i+1$ comparisons to the first interval $[0, \alpha]$. This proves that

$$
v_{k}(\alpha)=v_{\text {even }}(\alpha)=E_{i}(\alpha)
$$

for $\alpha \in\left[\alpha_{i}, \alpha_{i-1}\right]$, as desired.

We can similarly calculate the cross-over point when $k$ is odd to verify the other half of the lemma.

Note that the proof actually shows a stronger result, namely, for fixed $\alpha, v_{k}(\alpha)$ is equal to $v_{\text {even }}(\alpha)$ or $v_{\text {odd }}(\alpha)$ for $k$ large enough.

In the next section we need the following more precise statement of the lemma when $\alpha \in\left[\frac{1}{9}, \frac{1}{3}\right]:$ for all $k \geq 2$,

$$
v_{k}(\alpha)= \begin{cases}\frac{1+3 \alpha}{2} & \text { if } k \text { is even } \\ 1+\alpha & \text { if } k \text { is odd. }\end{cases}
$$

The following properties are easy to verify.

LEMMA 2. Let $k \geq 1$ be fixed.

1. For $\alpha$ in the range $\left[0, \frac{1}{2}\right]$, the functions $v_{k}(\alpha), v_{\mathrm{even}}(\alpha)$, and $v_{\mathrm{odd}}(\alpha)$ are continuous, increasing, and piecewise linear.

2. $v_{k}(0)=2^{-\lfloor k / 2\rfloor}$. Hence $v_{\text {even }}(0)=v_{\text {odd }}(0)=0$.

3. $v_{\text {odd }}(\alpha) \geq v_{\text {even }}(\alpha)$ with equality if and only if $\alpha=0$.

4. The Joint Case. Now consider the joint case where $I=J=[0,1]$, so $U_{k}(I, J)$ and $\sigma_{k}(I, J)$ are simply written $U_{k}$ and $\sigma_{k}$. If the resulting intervals after the first probe 
are $I^{\prime}$ and $J^{\prime}$, there are only two cases to consider: either $I^{\prime}$ and $J^{\prime}$ are disjoint (for which we can use the analysis of the previous section) or they are equal (which is a recursive situation). This observation implies that, for all $k \geq 1, U_{k}$ satisfies the recurrence

$$
U_{k}=\min _{0 \leq \alpha \leq 1 / 2}\left\{\max \left\{V_{k-1}(\alpha),(1-\alpha) U_{k-1}\right\}\right\}
$$

with $U_{0}=2$. By the definition of $\sigma_{k}$, the right-hand side is minimized by the choice $\alpha=\sigma_{k}$. Multiplying the equation by $2^{\lceil k / 2\rceil}$, we obtain the normalized form.

$$
u_{k}=\min _{0 \leq \alpha \leq 1 / 2}\left\{\max \left\{\varepsilon_{k} v_{k-1}(\alpha), \varepsilon_{k}(1-\alpha) u_{k-1}\right\}\right\}
$$

where $\varepsilon_{k}=2$ if $k$ is odd, otherwise $\varepsilon_{k}=1$.

Consider, with $k$ fixed, the graphs of $v_{k-1}(\alpha)$ and $(1-\alpha) u_{k-1}$. As $\alpha$ increases from 0 to $\frac{1}{2}$, both graphs intersect at most once since the latter decreases from $u_{k-1}$ (by (1), $\left.u_{k-1} \geq 2^{1-\lfloor(k-1) / 2\rfloor}\right)$ while the former, by Lemma 2 , increases from $2^{-\lfloor(k-1) / 2\rfloor}$. Recall that, by definition, $v_{k-1}\left(\frac{1}{2}\right)$ is the normalized uncertainty in the case of two disjoint intervals of equal size; thus $v_{k-1}\left(\frac{1}{2}\right)>\frac{1}{2} u_{k-1}$. Therefore, the two graphs intersect exactly once. The intersection is the point $\left(\sigma_{k}, u_{k} / \varepsilon_{k}\right)$. Thus we can rewrite (3) as

$$
u_{k}=\varepsilon_{k} v_{k-1}\left(\sigma_{k}\right)=\varepsilon_{k}\left(1-\sigma_{k}\right) u_{k-1} \quad(k \geq 1),
$$

where the base case is $u_{1}=2$ and $\sigma_{1}=\frac{1}{2}$. The values in Table 1 were computed by iterating this recurrence. Figure 2 illustrates this process.

The question naturally arises whether this process "converges" in a suitable sense, and, specifically, does $\left\{u_{k}\right\}$ converge? The answer is given in the next result.

THEOREM 3. The sequence $\left\{\left(\sigma_{k}, u_{k}\right)\right\}_{k=1}^{\infty}$ converges to $\left(\tilde{\sigma}_{\text {odd }}, \tilde{u}_{\text {odd }}\right):=\left(\frac{2}{7}, \frac{13}{7}\right)$ for $k$ odd, and to $\left(\tilde{\sigma}_{\mathrm{even}}, \tilde{u}_{\mathrm{even}}\right):=\left(\frac{3}{10}, \frac{13}{10}\right)$ for $k$ even.

PROOF. We first define a sequence $\left\{\tilde{\sigma}_{k}, \tilde{u}_{k}\right\}_{k \geq 2}$ and then relate it to our original sequence $\left\{\sigma_{k}, u_{k}\right\}_{k \geq 1}$. Let $f(x):=1+x$ and $g(x):=(1+3 x) / 2$. Let $\tilde{\sigma}_{2}:=\frac{1}{3}, \tilde{\sigma}_{3}:=\frac{5}{17}$, and, for $j \geq 1$, the following equations hold:

$$
\begin{aligned}
\tilde{u}_{2 j} & =f\left(\tilde{\sigma}_{2 j}\right)=\left(1-\tilde{\sigma}_{2 j}\right) \tilde{u}_{2 j-1}, \quad \text { and } \\
\tilde{u}_{2 j+1} & =2 g\left(\tilde{\sigma}_{2 j+1}\right)=2\left(1-\tilde{\sigma}_{2 j+1}\right) \tilde{u}_{2 j} .
\end{aligned}
$$

We now solve for $\sigma_{k}$ and $u_{k}$ : by the substitutions $\tilde{u}_{2 j-1} \rightarrow 2 g\left(\tilde{\sigma}_{2 j-1}\right)$ and $\tilde{u}_{2 j} \rightarrow$ $f\left(\tilde{\sigma}_{2 j}\right)$, we have

$$
\begin{aligned}
f\left(\tilde{\sigma}_{2 j}\right) & =2\left(1-\tilde{\sigma}_{2 j}\right) g\left(\tilde{\sigma}_{2 j-1}\right), \quad \text { and } \\
g\left(\tilde{\sigma}_{2 j+1}\right) & =\left(1-\tilde{\sigma}_{2 j+1}\right) f\left(\tilde{\sigma}_{2 j}\right) .
\end{aligned}
$$

Expanding the functions $f$ and $g$ and simplifying, we get

$$
\tilde{\sigma}_{2 j}=\frac{3 \tilde{\sigma}_{2 j-1}}{2+3 \tilde{\sigma}_{2 j-1}} \quad \text { and } \quad \tilde{\sigma}_{2 j+1}=\frac{1+2 \tilde{\sigma}_{2 j}}{5+2 \tilde{\sigma}_{2 j}}
$$




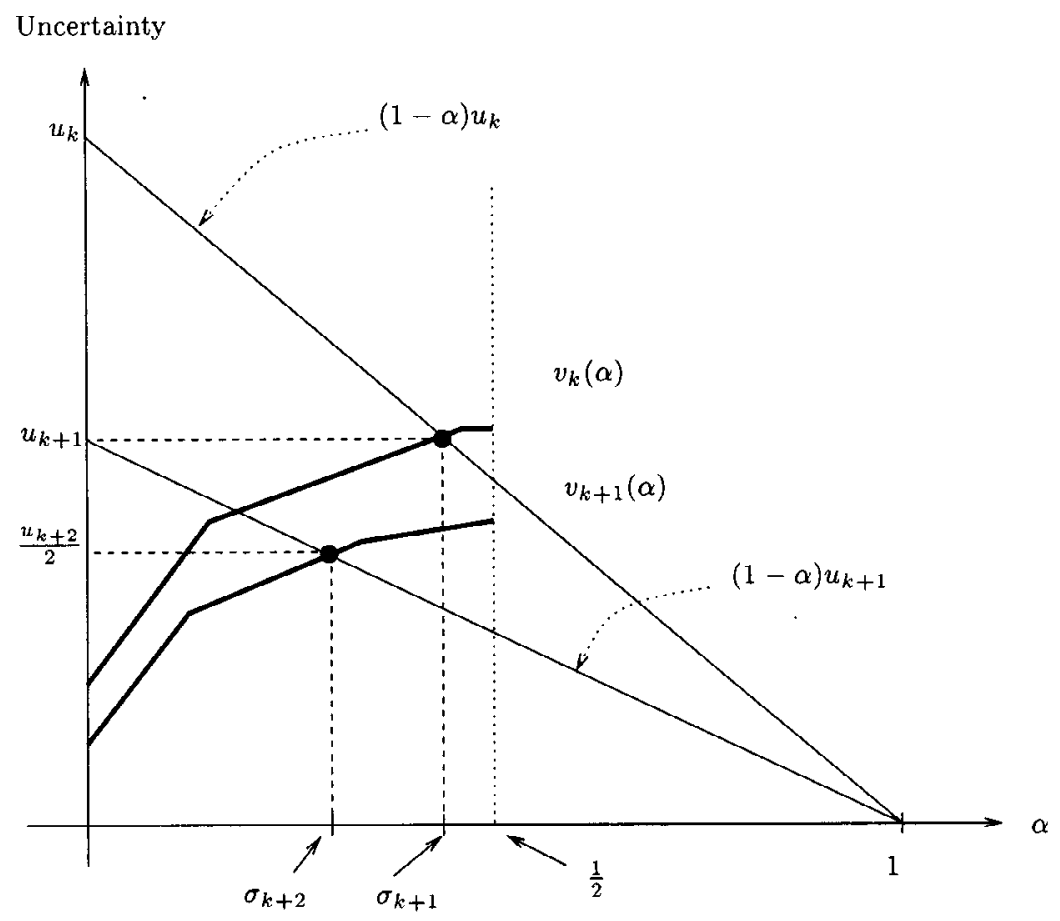

Fig. 2. Iterative process to find $u_{k+1}$ and $u_{k+2}$ from $u_{k}$ ( $k$ odd).

or

$$
\tilde{\sigma}_{2 j+2}=\frac{3+6 \tilde{\sigma}_{2 j}}{13+10 \tilde{\sigma}_{2 j}} \quad \text { and } \quad \tilde{\sigma}_{2 j+1}=\frac{2+9 \tilde{\sigma}_{2 j-1}}{10+21 \tilde{\sigma}_{2 j-1}} .
$$

These could be written as two independent iterative equations,

$$
\tilde{\sigma}_{2(j+1)}=F\left(\tilde{\sigma}_{2 j}\right) \quad \text { and } \quad \tilde{\sigma}_{2 j+1}=G\left(\tilde{\sigma}_{2 j-1}\right)
$$

where $F(x):=(3+6 x) /(13+10 x)$ and $G(x):=(2+9 x) /(10+21 x)$. Note that $F\left(\frac{3}{10}\right)=\frac{3}{10}$ and $G\left(\frac{2}{7}\right)=\frac{2}{7}$. Since $F$ is continuous and $0<F^{\prime}(x)<1$ for all $x \in\left[\frac{3}{10}, \frac{1}{3}\right]$, it easily follows that the sequence $\left\{\tilde{\sigma}_{2 j}\right\}_{j=1}^{\infty}$ converges monotonically decreasing to the fixed point $\frac{3}{10}$ since we started with $\tilde{\sigma}_{2}=\frac{1}{3}$. Similarly, with starting point $\tilde{\sigma}_{3}=\frac{5}{17}$, the sequence $\left\{\tilde{\sigma}_{2 j+1}\right\}_{j=1}^{\infty}$ converges monotonically decreasing to $\frac{2}{7}$. Figure 3 illustrates these two fixed points.

It remains to prove that $\sigma_{k}=\tilde{\sigma}_{k}$ for all $k \geq 2$. Note that, for $k \geq 2, g(x)=v_{k}(x)$ if $x \in\left[\frac{1}{9}, \frac{1}{3}\right]$ and $k$ is even (see (2)). Similarly $f(x)=v_{k}(x)$ if $x \in\left[\frac{1}{5}, \frac{1}{2}\right]$ and $k$ is odd. Therefore, (5) is equivalent to our original recurrence (4) provided $\tilde{\sigma}_{j} \in\left[\frac{1}{5}, \frac{1}{3}\right]$ whenever $j \geq 2, \tilde{\sigma}_{2}=\sigma_{2}$, and $\tilde{\sigma}_{3}=\sigma_{3}$. However, we established this provision in the previous paragraph. 


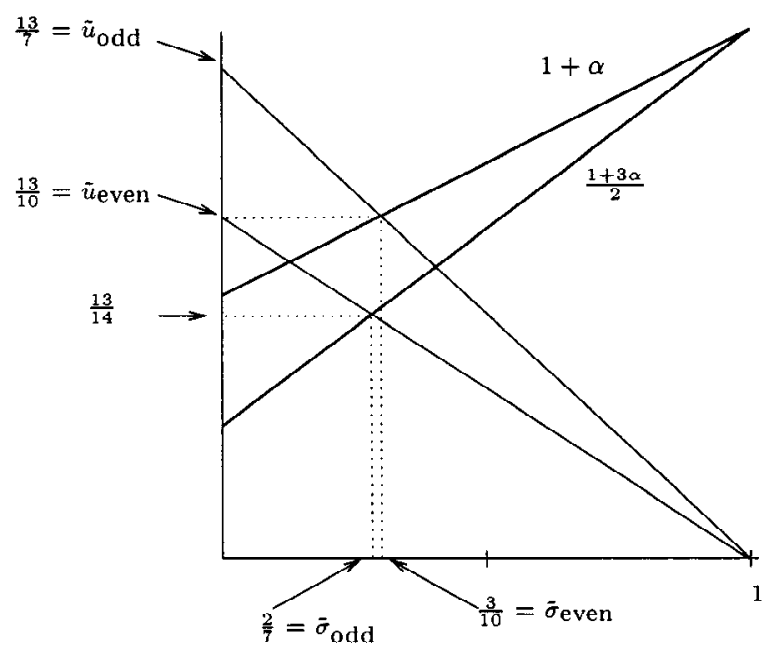

Fig. 3. The fixed point solution.

5. Remark. It is interesting to study the general case of $U_{k}(I, J)$ where $I$ and $J$ are arbitrary closed intervals in $\mathbb{R}$. For instance, if $|I|=|J|=1$, it is not hard to verify that

$$
1 \leq U_{1}(I, J) \leq 1.5 \text {. }
$$

More precisely, if $|I \cap J| \leq \frac{1}{2}$, then $U_{1}(I, J)=1.5$ and otherwise, $U_{1}(I, J)=2-|I \cap J|$. Similarly, we have

$$
\frac{2}{3} \leq U_{2}(I, J) \leq 1
$$

Furthermore, there is an obvious generalization to $n$ intervals $\left(I_{1}, \ldots, I_{n}\right)$ where each $I_{i}$ contains an unknown $x_{i}$. Another generalization is to define the uncertainty of $\left(I_{1}, \ldots, I_{n}\right)$ to be $\sum_{i} w_{i}\left|I_{i}\right|$, where $w_{i} \geq 0$ are specified weights.

\section{References}

[1] S. C. Feng and T. H. Hopp. A review of current geometric tolerancing theories and inspection data analysis algorithms. Technical Report NISTIR-4509, National Institute of Standards and Technology, U.S. Department of Commerce, Factory Automation Systems Division, Gaithersburg, MD 20899, February 1991.

[2] V. Srinivasan and H. B. Voelcker, editors. Dimensional Tolerancing and Metrology. CRTD, Vol. 27. The American Society of Mechanical Engineers, New York, 1993.

[3] C. K. Yap. Exact computational geometry and tolerancing metrology. In D. Avis and J. Bose, editors, Snapshots of Computational and Discrete Geometry, Vol. 3. McGill School of Computer Science, Technical Report No. SOCS-94.50, 1994. A volume dedicated to Godfried Toussaint.

[4] C. K. Yap and E.-C. Chang. Issues in the metrology of geometric tolerancing. In J.-P. Laumond and M. Overmars, editors, Algorithms for Robot Motion Planning and Manipulation, pages 393-400. A.K. Peters, Wellesley, Massachusetts, 1997. (Proc. 2nd Workshop on Algorithmic Foundations of Robotics (WAFR).) 